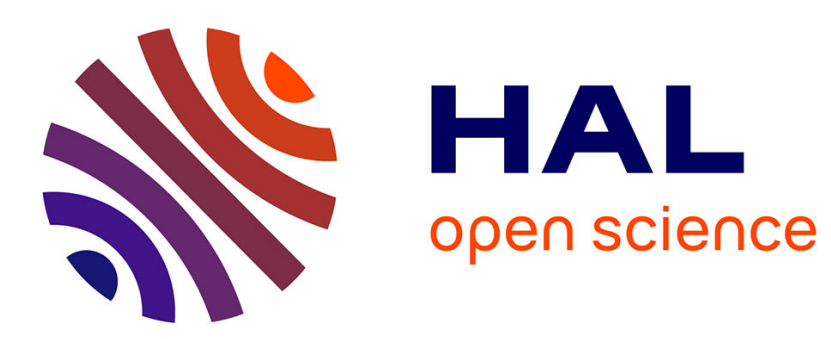

\title{
A neutron scattering study of the impurity effects on the one-dimensional antiferromagnet TMMC
}

\author{
J.P. Boucher, W.J. Fitzgerald, K. Knorr, C. Dupas, J.P. Renard
}

\section{To cite this version:}

J.P. Boucher, W.J. Fitzgerald, K. Knorr, C. Dupas, J.P. Renard. A neutron scattering study of the impurity effects on the one-dimensional antiferromagnet TMMC. Journal de Physique Lettres, 1978, 39 (7), pp.86-89. 10.1051/jphyslet:0197800390708600 . jpa-00231449

\section{HAL Id: jpa-00231449 https://hal.science/jpa-00231449}

Submitted on 1 Jan 1978

HAL is a multi-disciplinary open access archive for the deposit and dissemination of scientific research documents, whether they are published or not. The documents may come from teaching and research institutions in France or abroad, or from public or private research centers.
L'archive ouverte pluridisciplinaire HAL, est destinée au dépôt et à la diffusion de documents scientifiques de niveau recherche, publiés ou non, émanant des établissements d'enseignement et de recherche français ou étrangers, des laboratoires publics ou privés. 


\title{
A NEUTRON SCATTERING STUDY OF THE IMPURITY EFFECTS ON THE ONE-DIMENSIONAL ANTIFERROMAGNET TMMC
}

\author{
J. P. BOUCHER
}

Centre d'Etudes Nucléaires de Grenoble, DRF-G, 85 X, 38041 Grenoble Cedex, France

\section{W. J. FITZGERALD}

Institut Laue Langevin, 156 X, 38042 Grenoble Cedex, France

\section{K. KNORR}

Institut für Kristallographie, Tübingen, W. Germany

\section{DUPAS and J. P. RENARD}

Institut d'Electronique Fondamentale, Laboratoire associé au CNRS, Université Paris XI, Bât. 220, 91405 Orsay Cedex, France

(Reçu le 27 décembre 1977, accepté le 21 février 1978)

\begin{abstract}
Résumé. - Des mesures de diffraction quasi-élastique et inélastique de neutrons ont été faites sur des échantillons de TMMC dopé au cuivre, $\left(\mathrm{CD}_{3}\right)_{4} \mathrm{NMn}_{(1-x)} \mathrm{Cu}_{x} \mathrm{Cl}_{3}$ avec $x=0,0,03$ et 0,08 . La longueur de corrélation unidimensionnelle $\xi$ décroît en fonction de $x$ tandis que les ondes de spin s'élargissent. Le vecteur d'onde critique au-dessus duquel les magnons apparaissent est relié à $\xi$ par $Q_{\mathrm{c}} \simeq 2 a \xi^{-1} / \pi$ où $a$ est la distance entre spins. Ce résultat est en accord avec une précédente évaluation faite sur un échantillon de TMMC pur.
\end{abstract}

\begin{abstract}
Quasi-elastic and inelastic neutron scattering measurements have been performed on the magnetic system $\left(\mathrm{CD}_{3}\right)_{4} \mathrm{NMn}_{(1-x)} \mathrm{Cu}_{x} \mathrm{Cl}_{3}$ with $x=0.03$ and 0.08 . A decrease of the onedimensional correlation length $\xi$ with respect to pure TMMC is observed and discussed. Low $Q$ magnons have been studied at $4.2 \mathrm{~K}$ for $x=0,0.03$ and 0.08 . The dynamic critical value $Q_{\mathrm{c}}$ above which 1d magnons start to appear is related to the inverse correlation length $\kappa=a \xi^{-1} / \pi(a$ is the distance between spins) by $Q_{\mathrm{c}} \simeq 2 \kappa$, in agreement with other measurements as a function of temperature on pure TMMC.
\end{abstract}

1. Introduction. - As pointed out by Imry et al. [1] one-dimensional (1d) magnetic systems are rather sensitive to impurities. Indeed, impurities break the magnetic chains into nearly independent segments and restrict the development of $1 \mathrm{~d}$ spin correlations. An indirect result of this effect is the net decrease of the three-dimensional (3d) ordering temperature which has been observed in a series of quasi 1d magnetic compounds : $\left(\mathrm{CH}_{3}\right)_{4} \mathrm{NMnCl}_{3}$ (TMMC) [2], $\mathrm{CsNiF}_{3}$ [3]... At this time, the effect of impurities on the 1d correlation length $\xi$ has not yet been observed directly. TMMC seems to be a very good system to carry out such experiments because $\xi$ is rather large at low temperatures [4] : at $4.2 \mathrm{~K}, \xi$ includes about $30 \mathrm{Mn}$ spins and thus one can expect a net effect of impurities with atomic concentration of a few per cent.

Two kinds of experimental results will be reported in this paper : quasi-elastic and inelastic neutron scattering. The first experiment measured the correlation length $\xi$ and the second the 1d spin-wave excitations which will also be shown to be affected by impurities.

2. Sample preparation. - Single crystals of $\left(\mathrm{CD}_{3}\right)_{4} \mathrm{NMn}_{(1-x)} \mathrm{Cu}_{x} \mathrm{Cl}_{3}$ were prepared by slowly evaporating at room temperature a saturated solution of $\left(\mathrm{CD}_{3}\right) \mathrm{NCl}, \mathrm{MnCl}_{2} .4 \mathrm{D}_{2} \mathrm{O}$ and $\mathrm{CuCl}_{2} .2 \mathrm{D}_{2} \mathrm{O}$ in heavy water following the procedure previously 
used for growing $\left(\mathrm{CD}_{3}\right)_{4} \mathrm{NMnCl}_{3}$ [5]. The atomic concentration of $\mathrm{Cu}$ in the crystal was determined by the Laboratory of Microanalysis of CNRS, Thiais, France. For the present measurements, it would have been better to use diamagnetic impurities. However $\mathrm{Mg}$ and $\mathrm{Zn}$ do not replace $\mathrm{Mn}$ in TMMC and $\mathrm{Cd}$ doped crystals are rather difficult to grow with a homogeneous distribution. On the other hand the $\mathrm{Cu}-\mathrm{Mn}$ interaction is small compared to the Mn-Mn coupling and can be neglected to a first approximation. Three samples of about $1 \mathrm{mg}$ were used in the experiment with $x=0 ; 0.03$ and 0.08 , referred to in the following as pure, $3 \%$ and $8 \%$ samples.

3. Neutron experiments and results. - 3.1 QUASIELASTIC MEASUREMENTS. - The neutron scattering experiments were performed at the high flux beam reactor at the Institut Laue-Langevin (ILL) in Grenoble. For the quasi-elastic scattering measurements, the two-axis diffractometer D2 was used with

$$
\lambda=1.225 \AA \text {. }
$$

The collimations in front of the monochromator, the sample and the detector were $30^{\prime}, 20^{\prime}$ and $20^{\prime}$ respectively. Measurements were performed in the temperature range $1.7 \mathrm{~K}<T<80 \mathrm{~K}$ using an He-evaporation cryostat. Scans as a function of $q$, $(0.2 ; 0.2, q)$ were obtained for the $3 \%$ and $8 \%$ samples. Peaks have been observed around $q=1$, and as is explained in reference [4], these peaks belong to magnetic planes and are characteristic of $1 d$ shortrange order. The widths of the peaks observed for the $8 \%$ sample were larger than those of the $3 \%$ sample. Since the width of the magnetic part of these peaks is inversely proportional to $\xi$, the observed broadening establishes that the effect of impurities is actually to reduce the $1 \mathrm{~d}$ correlation length. We can try to compare quantitatively our quasi-elastic scattering measurements on impure crystals directly with the results of Birgeneau et al. on pure TMMC [4]. Following the procedure given in reference [4], we first substracted the parasite nuclear scattering contribution observed at relatively high temperature $(\sim 80 \mathrm{~K})$ from all the data. The resulting scattering was considered to be given as a function of $q$ by

$$
M(q)=\int L(q) R(q-s) \mathrm{d} s
$$

where $R(q)$ is the resolution function of the instrument and $L(q)$ represents the magnetic contribution to be studied. The function $L(q)$ was assumed to be Lorentzian

$$
L(q)=A /\left[\kappa^{2}+Q^{2}\right]
$$

where $Q=1-q$. The correlation length $\xi$ is related to $\kappa$ by

$$
\kappa=a \xi^{-1} / \pi
$$

where $a=3.247 \AA$ is the distance between adjacent spins in the same magnetic chain.

To analyse the data, a convolution procedure was used and a least squares fit was made, varying the parameters $A, \kappa$ and the peak position, until the best fit to the measured data $M(q)$ was obtained. The resulting values of $a \xi^{-1}$ for the $3 \%$ and $8 \%$ samples are plotted as a function of temperature in figure $1 a$, where the points can be compared directly to curve 1 . This curve corresponds to a pure classical Heisenberg system with $J=7.7 \mathrm{~K}$ and was shown to reproduce fairly well the experimental data on pure TMMC [4].
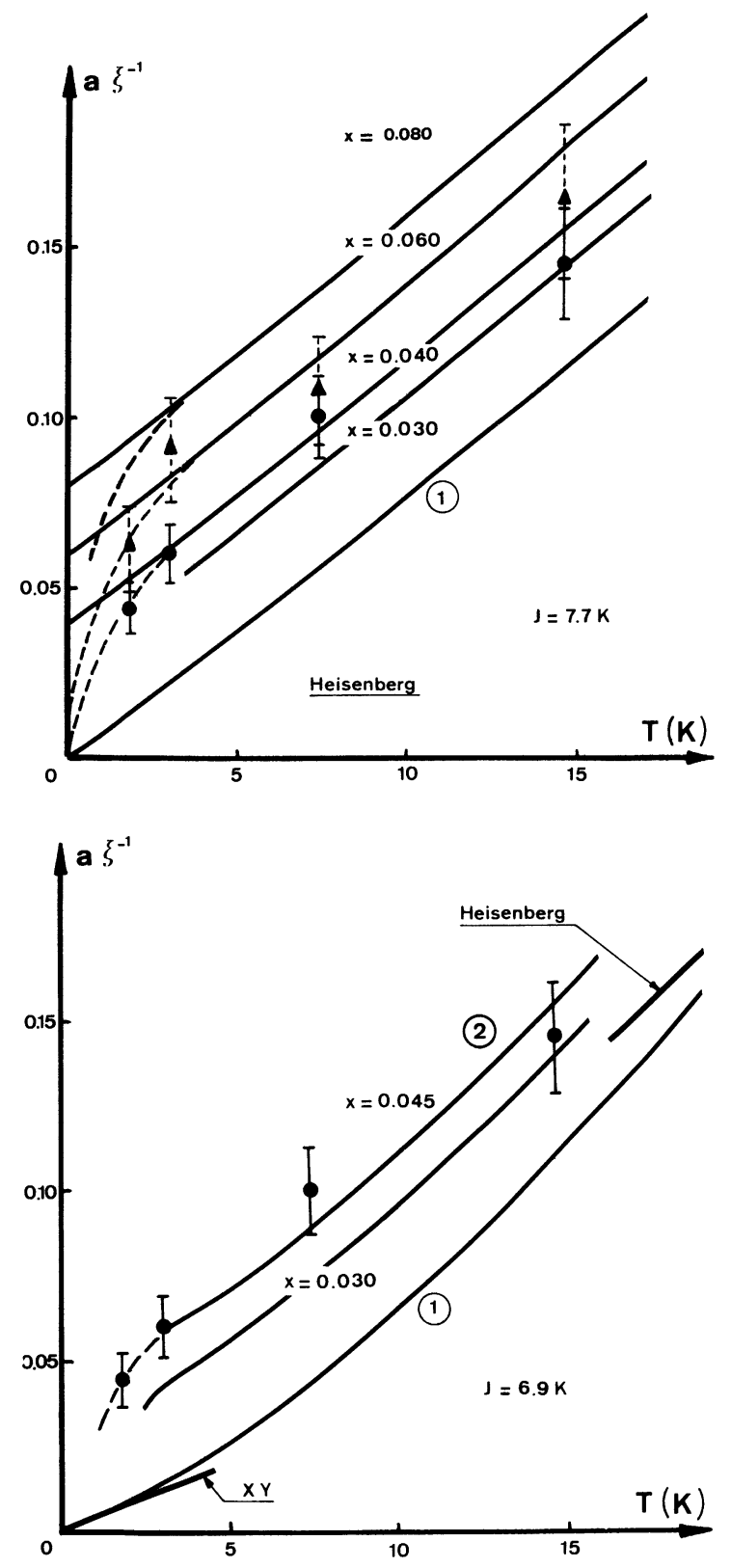

Fig. 1. - Experimental values of the reduced inverse correlation length $a \xi^{-1}$ versus temperature for two different impurity concentrations : $\bullet: 3 \% ; \Delta: 8 \%$. The lines are theoretical curves for different values $x$ of the impurity concentration and correspond to $a$ ) a Heisenberg model, $b$ ) a spin system which crosses over from Heisenberg to XY behaviour. 
3.2 INELASTIC SCATTERING MEASUREMENTS. - The inelastic scattering experiments were performed on the triple-axis spectrometer IN 2 at the ILL. Pyrolytic graphite was used as both the monochromator and analyser and the incoming neutrons had an energy of $3.14 \mathrm{THz}(13.7 \mathrm{meV})$. Higher-order contamination from the monochromator was reduced to approximately $10^{-4}$ of the first-order using a pyrolytic graphite filter. Measurements were made at $4.2 \mathrm{~K}$, using an evaporation cryostat, on a pure crystal of TMMC and on the $3 \%$ and $8 \%$ samples which have been used for the quasi-elastic scattering experiments presented above. Typical scans as a function of energy are shown on figure 2 for different wave-vectors $(0.2$, $0.2, q)$. Spin-wave excitations are clearly apparent for the three samples. The width $\delta$ of these magnons becomes broader as the concentration of impurity is increased. In so far as the magnon lifetime is determined by $\xi$, this behaviour also gives evidence of the shortening of the correlation length in random $1 \mathrm{~d}$ magnetic systems. For some values of $q$, a striking effect can be observed : the spin-wave excitations

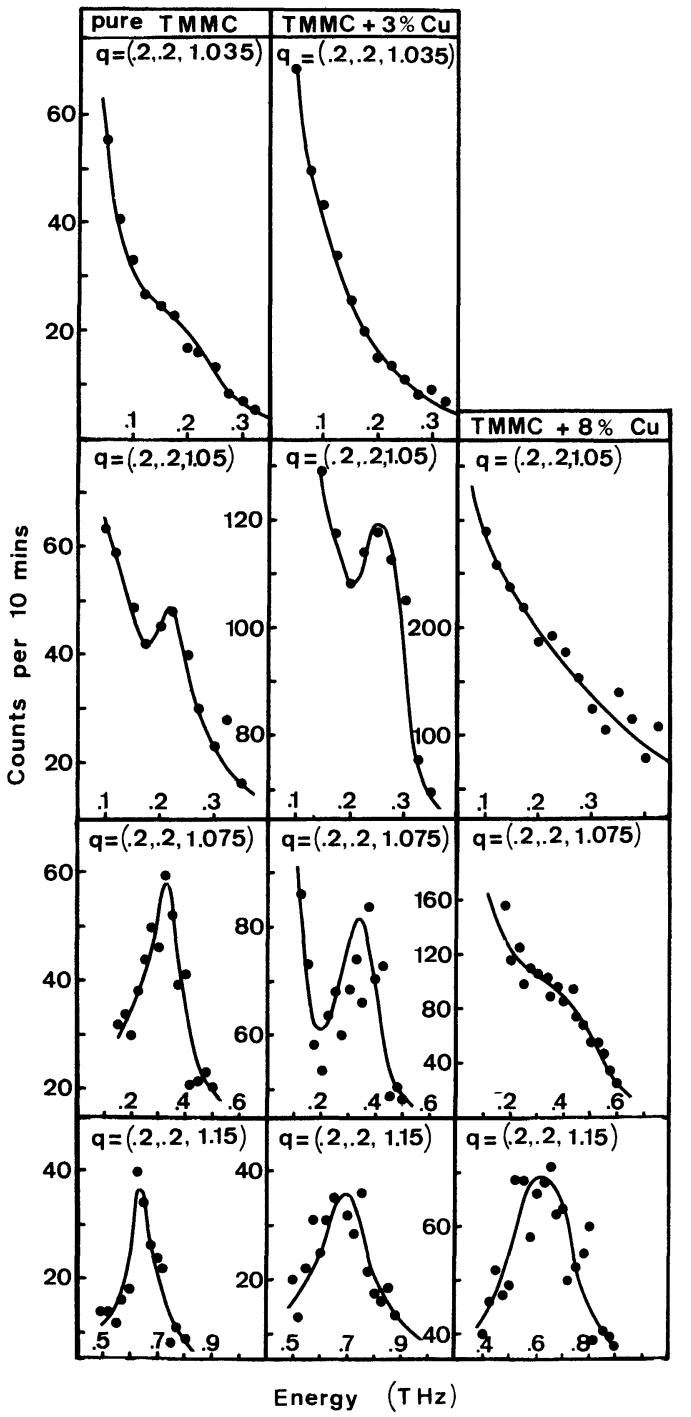

Frg. 2. - Typical inelastic scans obtained for the three crystals for different $q$ values. The curves are drawn as an aid to the eye. vanish as the impurity concentration is increased. Such an effect can be followed on figure 3 where for the same position of $q,(0.2 ; 0.2 ; 1.035)$ a scan for the pure sample is superimposed on a scan for the $3 \%$ sample. While a magnon is seen in the wing of the diffuse peak, no excitation is observed for the $3 \%$ sample. The same effect is also shown on figure 2 by comparing the $3 \%$ and $8 \%$ samples for the position $(0.2 ; 0.2 ; 1.05)$.

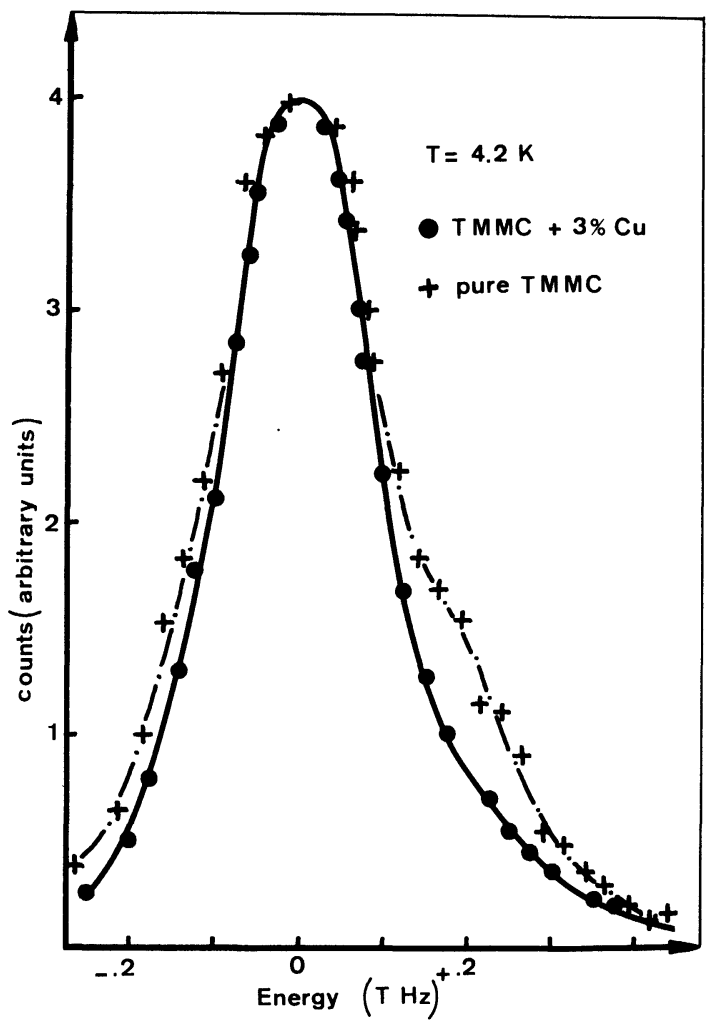

Fig. 3. - Inelastic scans at the position $(0.2 ; 0.2 ; 1.035)$ and $T=4.2 \mathrm{~K}$ for the pure and $3 \%$ samples.

4. Discussion. - The quasi-elastic scattering investigation of Birgeneau et al. on pure TMMC [4] was interpreted within the framework of the classical Heisenberg model. Curve 1 in figure $1 a$ corresponds to this model; the other curves were obtained from the theory developed by Thorpe for a random classical Heisenberg chain with a concentration $x$ of impurities [6]. The full lines are given for diamagnetic impurities while the dashed lines correspond to magnetic impurities. For this case, we choose for the $\mathrm{Mn}-\mathrm{Cu}$ coupling the value $J_{\mathrm{Mn}-\mathrm{Cu}} \simeq 1 \mathrm{~K}$ in accord with different evaluations [2] and we assumed the coupling between impurities to be negligible $J_{\mathrm{Cu}-\mathrm{Cu}}=0$. The effect of $J_{\mathrm{Mn}-\mathrm{Cu}}$ is important only below $T \sim 2 \mathrm{~K}$ where a net deviation from the curves for diamagnetic impurities can be seen. These theoretical curves account reasonably for the general behaviour of the experimental data. However, they disagree with the value of the concentration. The points relative to the $3 \%$ 
sample fit the curve for $x=0.04$ quite well while for the $8 \%$ sample, they agree approximately with the curve for $x=0.06$. The error bars indicated in figure 1 take into account statistical errors and the errors introduced by the fitting procedure and the inaccuracy of about $10 \%$ in the value of the impurity concentration. Within these experimental errors the agreement between theory and experiment is certainly reasonable for the $3 \%$ sample. However for the $8 \%$ sample the disagreement is greater. But it can be argued that for such a strong concentration of $\mathrm{Cu}$, the impurities may be arranged not quite randomly in the crystal : clusters of $\mathrm{Cu}$ atoms can exist yielding an effective concentration smaller than the value given by chemical analysis.

It must be pointed out that the interpretation of the low temperature magnetic properties of TMMC within the Heisenberg model seems questionable. Recent experimental studies have shown that TMMC is better understood in terms of an XY system. For instance, the effect of diamagnetic impurities [2] or the effect of a magnetic field [7] on the $3 \mathrm{~d}$ ordering temperature $T_{\mathrm{N}}$ can be explained only within the XY model. This assumption is also supported by a calculation of D. Hone and A. Pires [8] where it is shown that the dipolar interactions between electron spins in TMMC lead to a cross over from Heisenberg to $\mathrm{XY}$ behaviour starting at temperatures as high as $20 \mathrm{~K}$. Curve 1 in figure $1 b$, which has been taken from ref. [8] where $J \sim 6.9 \mathrm{~K}$, shows the cross over of $\xi^{-1}$ from Heisenberg to XY behaviour for a pure crystal. Except at low temperature $(T \lesssim 2 \mathrm{~K})$ where the effect of the host-impurity coupling is important, the value of $\xi^{-1}(x)$ for a given concentration $x$ can be obtained from the value $\xi^{-1}(0)$ for a pure system by the relation, valid to first order in $x$,

$$
a \xi^{-1}(x)=x+a \xi^{-1}(0)
$$

Curve 2 in figure $1 b$ which fits well the data for the $3 \%$ sample corresponds to the value $x=0.045$.
In this model, the disagreement between theory and experiment is important and not explained.

At least for the small values of $Q$ that we have explored, the dispersion of the spin-wave excitation is about the same for the three samples and fits within experimental error the theoretical curve for the classical Heisenberg model with $J \sim 6.6 \mathrm{~K}$ [5]. From figure 2 we can determine approximately the critical value $Q_{\mathrm{c}}$ at which the magnetic excitations vanish, at $4.2 \mathrm{~K}$, for the different concentrations of impurities (cf. Table I). The corresponding values of $\kappa$ can be obtained from the quasi-elastic scattering experiments. From figure $1 a$ we obtain the values given in table I and one would estimate the relation between the dynamic critical wave-vector $Q_{\mathrm{c}}$ and the inverse $1 \mathrm{~d}$ correlation length $\kappa$ to be $Q_{\mathrm{c}} \simeq 2 \kappa$. This relation is in reasonable agreement with the determination of Shirane $e t$ al. who proposed $Q_{\mathrm{c}} \sim 1.5 \kappa$ by analysing the very low $Q$ dynamics in a pure sample of TMMC [9]. As is pointed out in reference [9], this result does not agree with any existing theories.

Finally, a remark should be made concerning the choice of values for $J$. As one can see, by comparing the Heisenberg curve of figure $1 b$ with $1 a$ the results are very sensitive to $J$. The value $J \simeq 7.7 \mathrm{~K}$ given in reference [5] and used in figure $1 a$ seems to be much too large compared to the values obtained from many other determinations (magnetic susceptibility, specific heat, magnetization...) which are all consistent with $J=6.6 \pm 0.5 \mathrm{~K}$.

\section{TABLE I}

Dynamic critical vector $Q_{\mathrm{c}}$ and corresponding inverse correlation length $\kappa$ as a function of impurity concentration $x$.

$\begin{array}{lcc}x & 3 \% & 8 \% \\ \overline{Q_{\mathrm{c}}} & \sim \overline{0} & \overline{0} \\ \kappa & 0.04 & \sim 0.06 \\ & & 0.03\end{array}$

\section{References}

[1] Imry, Y., Montano, P. A. and Hone, D., Phys. Rev. B 12 (1975) 253. Notice that the effect of impurities was first studied in one-dimensional conductors. See, for instance, Bloch, A. N. and Varma, C. M., J. Phys. C 6 (1973) 1849 and references herein.

[2] Dupas, C. and Renard, J. P., Phys. Lett. 55A (1975) 181.

Dupas, C. and Renard, J. P., Physica 86-88B (1977) 705

[3] Steiner, M. and Axmann, A., Solid State Commun. 19 (1976) 115.

[4] Birgeneau, R. J., Dingle, R., Hutchings, M. T., Shirane, G. and Holt, S. L., Phys. Rev. Lett. 26 (1971) 718.
[5] Hutchings, M. T., Shirane, G., Birgeneau, R. J. and Holt, S. L., Phys. Rev. B 5 (1972) 1999.

[6] THORPe, M. F., J. Physique 36 (1975) 1177.

Tonegawa, T., Shima, H. and Pincus, P., Phys. Rev. B 11 (1975) 4683.

[7] Borsa, F., Boucher, J. P. and Villain, J., 23rd Conf. on Magnetism and Magnetic Materials, Minneapolis, Nov. 1977.

[8] Hone, D. and Pires, A., Phys. Rev. B 15 (1977) 323.

[9] ShIRANe, G. and Birgeneau, R. J., Physica 86-88B (1977) 639. 